\title{
LightProbe: A Digital Ultrasound Probe for Software-Defined Ultrafast Imaging
}

\section{Journal Article}

\section{Author(s):}

Hager, Pascal Alexander; Benini, Luca (D)

Publication date:

2019-04

Permanent link:

https://doi.org/10.3929/ethz-b-000335161

Rights / license:

In Copyright - Non-Commercial Use Permitted

Originally published in:

IEEE Transactions on Ultrasonics, Ferroelectrics, and Frequency Control 66(4), https://doi.org/10.1109/TUFFC.2019.2898007 


\title{
LightProbe: A Digital Ultrasound Probe for Software-Defined Ultrafast Imaging
}

\author{
Pascal Alexander Hager, Student Member, IEEE, and Luca Benini, Fellow, IEEE
}

\begin{abstract}
Digital ultrasound probes integrate the analog frontend in the housing of the probe handle and provide a digital interface instead of requiring an expensive coaxial cable harness to connect. Current digital probes target the portable market and perform the bulk of the processing (beamforming) on the probe, which enables the probe to be connected to commodity devices such as tablets or smartphones running an ultrasound app to display the image and control the probe. Thermal constraints limit the number of front-end channels as well as the complexity of the processing. This prevents current digital probes to support advanced modalities such as Vector Flow or Elastography requiring high-frame rate (HFR) imaging.

In this paper, we present LIGHTPROBE, a digital ultrasound probe, which integrates a 64-channel $100 \mathrm{Vpp}$ TX/RX frontend including analog-to-digital conversion (up to 32.5 MS/s @ 12 bit), and is equipped with an optical high-speed link $(26.4 \mathrm{~Gb} / \mathrm{s})$ providing sustainable raw samples access to all channels, which allows the processing to be performed on the connected device without thermal power constraints. By connecting the probe to a GPU-equipped PC, we demonstrate the flexibility of softwaredefined B-mode imaging using conventional and ultrafast methods. We achieve plane-wave and synthetic aperture imaging with frame-rates from $30 \mathrm{~Hz}$ up to $500 \mathrm{~Hz}$ consuming between $5.6 \mathrm{~W}$ and $10.7 \mathrm{~W}$. By using a combination of power and thermal management techniques, we demonstrate that the probe can remain within operating temperature limits even without active cooling, while never having to turn the probe off for cooling hence providing a consistent Quality of Service for the operator.
\end{abstract}

Index Terms-ultrasound imaging, medical imaging, optical high-speed link, digital ultrasound probe, ultrasound front-end, system implementation, thermal aware QoS

\section{INTRODUCTION}

Traditional ultrasound imaging system operate with a passive transducer probe, where the piezoelements in the probe are connected directly over an analog coaxial cable harness to a backend system. The backend system contains the analog front-end to send ultrasound pulses and to receive echos, as well as the digital processing unit to compute the output image.

Today, two trends in ultrasound system design can be observed: On one hand, towards software-defined ultrasound imaging, where the entire signal processing is performed in software [1]. In the past, general processing units did not provide enough compute power and custom hardware solutions were required to compute the image from the raw probe signals. Today, the image formation is increasingly performed in software on graphics processing units (GPUs)

P. Hager and L. Benini are with the Integrated Systems Laboratory at ETH Zürich, Switzerland, \{phager, benini\} @iis.ee.ethz.ch

L. Benini is also with the Electrical, Electronic, and Information Engineering Department of the University of Bologna, Italy, \{luca.benini\} @unibo.it
[2]-[4] and multicore processors [5]-[7]. These new system architectures have not only enabled new imaging modalities (Ultrafast Imaging [8], [9], Vector Flow Imaging [10], [11], Elastography [12]), but have also reduced system cost, as no ultrasound-specific hardware is needed for processing. The Aixplorer (Supersonic Imaging) or the DiPhAS (Fraunhofer IBMT) are two examples of such software-defined systems. Both systems adopt the ultrafast system architecture paradigm [8], [13], which proposes that the raw digitized data should be directly sent to a GPU for processing. The required highbandwidth link to transfer raw data from the analog-to-digital converters (ADCs) into the memory of the GPU is one of the main challenges for software-defined systems: A 256-channel system sampling all channels simultaneously with $12 \mathrm{~b}$ and $30 \mathrm{MS} / \mathrm{s}$ produces raw data at a rate of almost $100 \mathrm{~Gb} / \mathrm{s}$.

The second system trend is towards digital ultrasound probes: These probes no longer need an expensive and bulky analog cable to connect, but provide a wired or wireless digital interface such as USB or Wi-Fi to connect to a commodity device such as a smartphone, tablet or PC, which runs an ultrasound software application to control the probe and to display the image. To provide this connectivity, the digital probe embeds the analog front-end in the probe housing and performs at least part if not all of the digital processing on the probe [14]-[16]. On-probe processing is inevitable as already a 16-channel probe sampling with $12 \mathrm{~b}$ at $30 \mathrm{MS} / \mathrm{s}$ produces data at a rate of $5.8 \mathrm{~Gb} / \mathrm{s}$, which already exceeds what standard links, such as USB 2.0 (max. $0.48 \mathrm{~Gb} / \mathrm{s})$, USB 3.0: $(5 \mathrm{~Gb} / \mathrm{s})$ or Wi-Fi 802.11n (max. $0.6 \mathrm{~Gb} / \mathrm{s}$ ) can provide.

Digital probes connected to a smartphone or tablet running an ultrasound app are widely used to build portable systems such as the MobiUS PE System (MobiSante, Redmond, USA), the Philips Visiq \& Lumify (Philips Healthcare, NL) [16], [17], the Clarius Handheld Wireless Scanners (Clarius, CAN) [18] or the iQ (Butterfly Network, USA). While these digital probes provide already good imaging quality for standard B-mode, they do not (yet) support the more demanding modalities such as Ultrafast Imaging, Vector Flow or Elastography.

The main reason for this is that digital probes are thermallylimited devices [15], meaning that the amount of power that can be dissipated within the probe is limited, as otherwise the probe would become too hot. The surface temperature of a device in direct contact with the patient must be kept below $43^{\circ} \mathrm{C}$ in order to comply with medical safety regulations (IEC 60601-1 [19]). This thermal limitation restricts the number of receive channels and the complexity of the processing feasible to implement on a digital probe: Even tough the power consumption per receive channel has decreased significantly in 
the past years, from $100 \mathrm{~mW}$ down to $40 \mathrm{~mW}$ [20], [21], a 64channel probe still consumes $2.6 \mathrm{~W}$ in the receive path alone. Thus, digital probes typically operate with no more than 16 receive channels (amplifier, ADC) and use time-multiplexing [14] or analog pre-beamforming [22] to support larger transducer arrays and to have enough thermal budget left for onprobe processing. However, to support ultrafast modalities, all transducer channels should be sampled simultaneously and more complex processing is required. Fitting both into the thermal budget of a probe cannot easily be achieved.

To resolve this issues, we propose a novel ultrasound system architecture, which relies on an ultrafast digital probe that samples all channels simultaneously and uses a high-speed digital link to transfer the raw channel data to an off-the-shelf PC subsystem, where the processing is performed without thermal power constraints.

This architecture combines the flexibility of softwaredefined systems and the cost-efficiency of digital probes. We expect such an architecture to come with significant cost savings as it requires only a minimal amount of ultrasoundspecific hardware, i.e., just the probe and a refurbished housing for the PC with a console keyboard. Also, the expensive coaxial analog cable harness is no longer needed, which itself has a significant contribution to the price of the system. While it has been demonstrated that tablets and smartphones [23] could provide sufficient compute power on their embedded GPUs for software beamforming, these devices today do not yet provide sufficient external I/O bandwidth to sustainably receive the raw data. We therefore focus on a PC-based processing system for the time being.

The challenge to build an ultrafast digital probe is to include a high number of front-end channels (64 or more) and a highspeed $(>10 \mathrm{~Gb} / \mathrm{s})$ link into the probe within thermal limits.

In this work, we demonstrate with a fully-functional ultrafast digital probe prototype (LIGHTPROBE) that this challenge can be addressed by combining the following three approaches elaborated in detail in Sec. II: First, we equip the probe with a fiber optical link to provide sufficient link bandwidth $(>25 \mathrm{~Gb} / \mathrm{s})$. Second, we reduce the average power consumption for normal B-mode imaging by exploiting the ultrafast imaging capabilities of the probe. Third, we develop a thermal management solution that enables high-performance operation modes with a power consumption exceeding the passive cooling capabilities of the probe. Part of this work builds on a concept we initially presented in [24] and the ULTRALIGHT system we described in [25], where we have shown a first overview of this work and focused on how a complete imaging system can be built using LIGHTPROBE and a standard PC equipped with a GPU.

In this paper, we focus on the probe (LightProBE), provide hardware details, perform extensive quality and performance evaluation on different imaging methods and introduce the thermal management needed to safely operate such a device. The contributions of this work are:

1) A hand-held transducer probe hardware prototype integrating an entire 64-channel ultrasound front-end and equipped with an optical high-speed link $(26.4 \mathrm{~Gb} / \mathrm{s})$ to connect to a PC for software-defined ultrasound imaging.
2) We demonstrate that, even with only passive cooling, a digital probe can support continuous plane-wave imaging with a frame rate of $47 \mathrm{~Hz}$ indefinitely.

3) We also demonstrate how high-frame-rate imaging (200$500 \mathrm{fps}$ ) can be safely supported in a digital probe, despite high peak power consumption $(10.7 \mathrm{~W})$ and high link bandwidth requirements $(15.36 \mathrm{~Gb} / \mathrm{s})$.

Next, we elaborate the main design decisions (Sec. II) before we outline the system architecture (Sec. III) and implementation (Sec. IV). We show extensive measurements (Section VI-VII) to demonstrate our system works, produces good images and does not overheat. Finally, we discuss our the results (Sec. VIII) and close with conclusions.

\section{Designing An Ultrafast Probe}

\section{A. Avoid Heat from Processing}

High-frame-rate (HRF) imaging is extremely compute intensive. [9] estimates several TFLOP/s are required for gray-scale compounded plane-wave ultrafast imaging. The first problem to solve when designing a digital probe for ultrafast imaging is to move the power-hungry processing from the probe into the PC, where there are no thermal power constraints. Current state-of-the-art GPUs like the Nvidia Tesla V100, provide sufficient processing power (15 TFLOP/s) and I/O bandwidth $(1.2 \mathrm{~Tb} / \mathrm{s})$ for this task.

This however requires the digital raw data from all channels to be transfer from the probe to the PC: A 64-channel probe sampling with $12 \mathrm{~b}$ at $32.5 \mathrm{MS} / \mathrm{s}$ produces a datastream of $24.96 \mathrm{~Gb} / \mathrm{s}$. This rate is not yet supported with current common interfaces like USB $3.1(10 \mathrm{~Gb} / \mathrm{s})$ and USB $3.2(20 \mathrm{~Gb} / \mathrm{s})$. USB Type $\mathrm{C}$ allows simplex datarates up to $4 \times 10 \mathrm{~Gb} / \mathrm{s}$, along with an auxiliary USB 2.0 and supply power. However, currently available copper-based USB Type $\mathrm{C}$ cables providing these rates are restricted to less than $1 \mathrm{~m}$ length, which would make the handling of the probe uncomfortable for the user.

Higher data-rate are possible with optical links, which can be easily scaled to even higher data-rates to support even more channels. Optical links also impose no practical limits on the length. Optical point-to-points links providing $40 \mathrm{~Gb} / \mathrm{s}$ or even $100 \mathrm{~Gb} / \mathrm{s}$ are widely used in data centers today, as they provide a higher throughput at lower energy consumption compared to copper-based solutions [26]. We thus decided to equip our probe with a link based on the Quad Small Formfactor Pluggable (QSFP) standard, which allows datarates of up to $40 \mathrm{~Gb} / \mathrm{s}$ over a optical fiber, requires as little as $1 \mathrm{~W}$, and is readily available at low cost (100-200 \$ USD).

Being able to stream raw data from the probe also allows buffering entire acquisitions sequences (several GB) for offline processing, as high-bandwidth high-capacity memory is easily provided in the connecting PC system. Providing this buffering in the space and power constraints of the probe is not easily possible. Offline processing is interesting for applications, where real-time output is not needed, i.e. for Vector Flow Imaging, where the output can be computed offline, before a slow-motion playback is provided to the operator. 


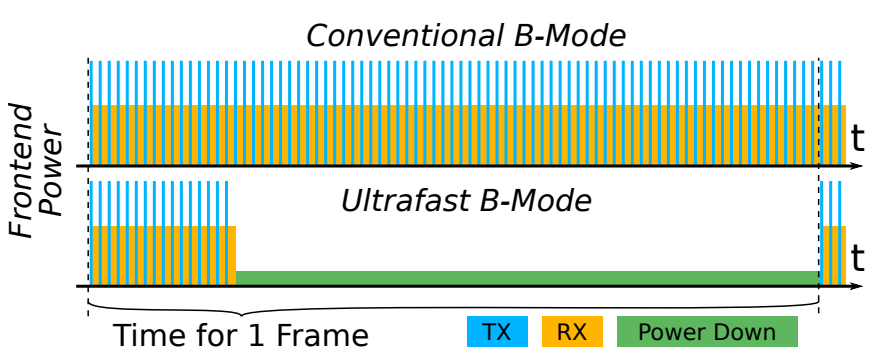

Fig. 1. Ultrasound front-end power consumption for B-Mode imaging at $25 \mathrm{~Hz}$ : Conventional imaging may use the whole frame time period to scan the entire field-of-view (FoV). Ultrafast methods capture the entire FoV with much fewer shots. This drastically reduces the required energy to capture a frame as less energy-intensive transmissions are required and the front-end can be powered down for a large share of the time.

\section{B. Exploit Ultrafast Imaging to Reduce Energy Consumption}

Plane-wave imaging allows to obtain a comparable B-mode image [27] with much fewer shots than what is required for conventional line-by-line imaging: [28] reports that a good image can already be produced with 21 plane-waves. Considering plane-wave imaging with a pulse repetition frequency (PRF) of $5 \mathrm{kHz}$, a complete frame can be captured in a short pulse burst taking only $4.2 \mathrm{~ms}$. Assuming a target frame-rate of $25 \mathrm{~Hz}$, the front-end can be turned off for almost $90 \%$ of the time. Lineby-line imaging requires many more shots to sequentially scan the entire field-of-view (FoV). As this scanning process may take as long as the entire frame period, the front-end may not be turned off. As illustrated in Fig. 1, performing B-mode imaging with plane-wave isonification allows to significantly reduce the average front-end power consumption. This relaxes the thermal budget of the probe and allows placing more channels in the digital probe. To materialize these power savings, the front-end hardware of the probe must support power down modes with fast transition times and low power sleep states. Our probe is optimized for this (Sec. IV-B) and equipped with a dynamic power management system (Sec. V-A), which activates the best possible power mode at all times. The achieved energy savings and quality impact will be quantified in Sec. VII.

\section{Boost Mode for High-Frame Rate Imaging}

Performing normal rate B-Mode imaging with ultrafast methods has allowed us to reduce the average power consumption of the front-end sufficiently to equip the probe with a front-end that allows simultaneous sampling of all transducer channels. This enables high-frame-rate (HFR) imaging required for Vector Flow or Elastograhpy. However, operating the probe continuously in HFR imaging will result in a power consumption which no longer allows to keep the probe's surface temperature within regulatory requirements with passive cooling only. On the other hand, many HFR applications not necessarily require continuous operation. A vector flow slow motion video or elastography map only requires a brief acquisition sequence taking no longer than a few seconds to capture. One option is to operate the probe in a dual-mode operation, where the probe is operated by default in normal-rate B-mode imaging. With this mode the right position of the probe can be found before activating

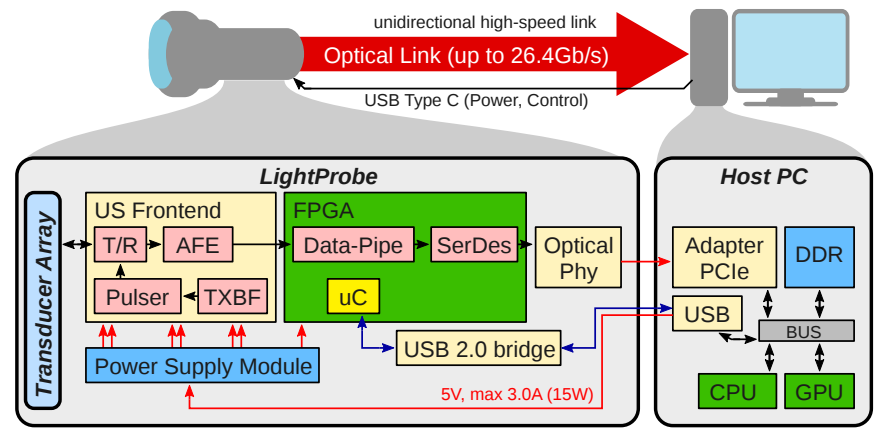

Fig. 2. System block diagram of an ultrasound imaging system using the LightProbe. The LightProbe connects to a host system over a unidirectional digital optical link. Control (USB 2.0) and power (max. $15 \mathrm{~W}$ ) are provided over a single USB Type C. The PC is equipped with an adapter to move the raw data received over the optical link into the main memory.

an advanced mode requiring HFR imaging. By dynamically controlling the imaging parameters (e.g. frame-rate) of the normal mode the probe can be kept at a temperature, which allows that the HFR boost-mode to be activated for a certain duration and periodicity indefinitely, without ever having to turn the system off for cooling.

\section{System ARChiteCTURE}

The system architecture of our software-defined imaging system using a digital probe is shown in Fig. 2: It consists of the LightPRoBe connected to a host PC. While the probe is mainly responsible for the raw signal acquisition, including the ultrasound isonification, analog-to-digital conversion and data transmission, the host $\mathrm{PC}$ runs the ultrasound imaging application, which controls the probe and performs the processing. Tbl. I summarizes the hardware specifications of the probe: The probe integrates the piezo-electric transducer array along with a 64-channel ultrasound front-end. It features a Field-Programmable-Gate-Array (FPGA) for control, data aggregation and processing, as well as multiple communication interfaces and a power supply module. The probe connects to the host PC over a unidirectional optical link for raw data transfer, and a USB Type C cable to provide power (max. $15 \mathrm{~W}$ ) and a communication channel to control the probe. On the PC, an adapter-card provides the optical link connector.

The probe could also be connected to portable devices such as tablets or smartphones by using the USB connector or the integrated WLAN module. For these cases, the required data bandwidth on the digital link has to be reduced with on-probe processing, which is not discussed in this work.

\section{LightProbe Design}

The main goal when designing LightPRoBE was to realize a probe that could be considered truly hand held. To achieve a very compact design, the LIGHTPROBE is implemented with a specially designed PCB stack (Fig. 3). The stack has a size of $220 \times 25 \times 45 \mathrm{~mm}^{3}$, consists of two 32-channel TX boards, a high-voltage supply module, an interface board and a motherboard. The latter carries an FPGA module and provides the RX stage and the optical connectors. The following subsections describe the different subsystems of the probe in detail. 


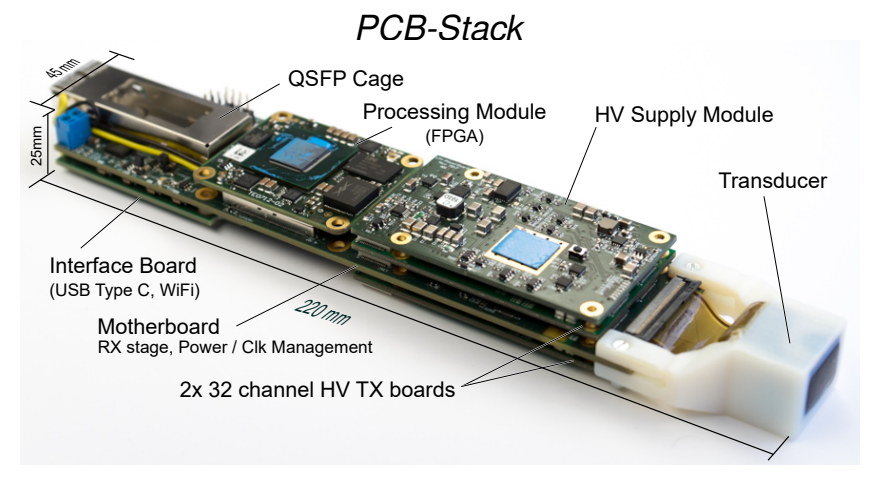

\section{LIGHTPROBE in Housing with Cables}

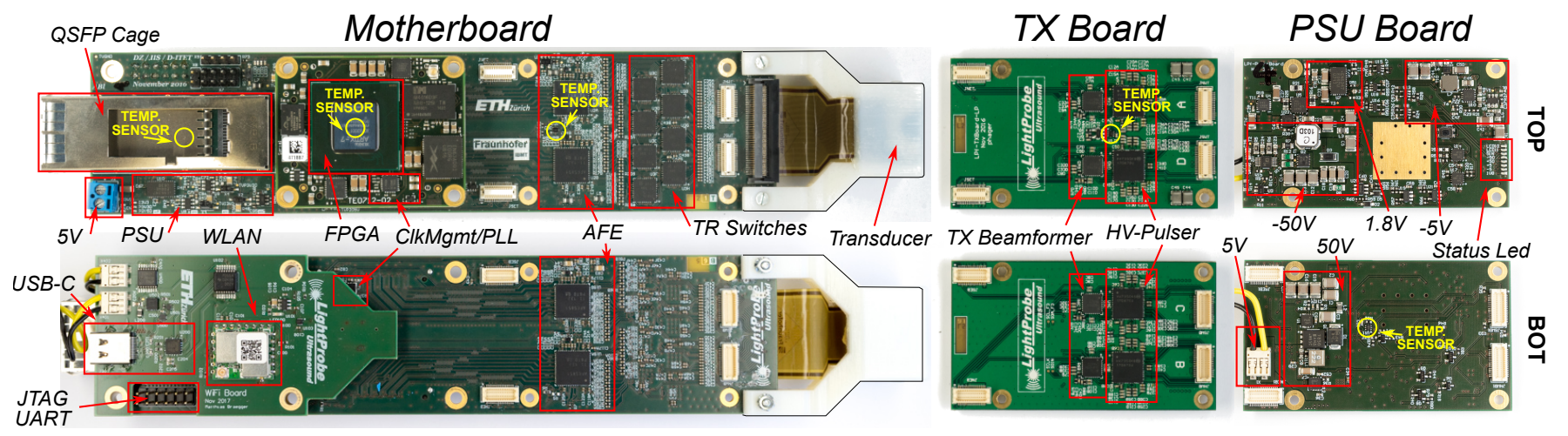

Fig. 3. The LightProbe PCBs stack with and without the housing (ToP) and the individual PCBs (BotTOM). Two cables (USB and QSFP) are required to connect the probe to the PC for ultrafast imaging: USB for power and control, and the fiber (QSFP) for data. The probe is also equipped with WLAN (not used in this work). Temperature sensors (shown in yellow) are placed to monitor the temperature at critical locations close to all major heat sources.

TABLE I

LightPROBE HARDWARE SPECIFICATIONS

\begin{tabular}{ll}
\hline System & - 64 RX/TX channels with size of $25 \times 45 \times 220 \mathrm{~mm}^{3}$ \\
& - Xilinx Artix-7 FPGA (XC7A200T-2), 1 GB DDR3 Memory \\
\hline Interfaces & - up to $26.4 \mathrm{~Gb} / \mathrm{s}$ optical interface over QSFP \\
& - USB Type C (Power max. 15 W, USB 2.0 for control, UART) \\
& - Wireless LAN IEEE $802.11 \mathrm{~b} / \mathrm{g} / \mathrm{n}$ (not used in this work) \\
\hline TX stage & - per channel configurable bipolar 64 pulse sequences \\
& - 100 Vpp, 0.625 to $20 \mathrm{MHz}$ pulse frequency \\
& - Delay Range $102.4 \mu \mathrm{s}$, Delay Resolution $0.78 \mathrm{~ns}$ \\
\hline RX stage & - Bandwidth $14 \mathrm{MHz}$ (AAF), $12 \mathrm{~b}$ up to $32.5 \mathrm{MS} / \mathrm{s} \mathrm{ADC}$ \\
& - digitally controlled, variable gain amplification: -5 to $31 \mathrm{~dB}$ \\
\hline
\end{tabular}

\section{A. Ultrasound Transducer Array}

The LightPRoBe is currently equipped with a custom 64element $4 \mathrm{MHz}$ linear phased-array transducer fabricated by Fraunhofer IBMT (Germany). For details about the transducer see [25]. Since the transducer is connected to the front-end with a plug, it can be easily replaced. We support transducer arrays with up to 64-elements without the need for multiplexing. To support transducers with up to 256 elements the PCB stack could easily be extended with a 4:1 multiplexer board.

\section{B. Ultrasound Front-End}

The ultrasound front-end (Fig. 2) supports 64 channels and is built from discrete components. It contains the hardware to emit ultrasonic pulses, as well as to amplify and digitize the received echoes: The pulse wave-forms are generated by a TX-Beamformer (TXBF) and converted to high-voltage signals required to excite the piezoelectric transducer elements by a $H V$-Pulser. On the receive path, a TX/RX-Switch (T/R) blocks the high-voltage pulses to protect the sensitive analog receive front-end (AFE), which contains the amplifiers and the analogto-digital converters (ADC). The components for the front-end are chosen to provide low power consumption, good and fast power down modes, and to enable a compact implementation:

For the TX pulser, an integrated 8-channel chip (HV7350, Microchip) is used, which employs a special direct-coupling topology for the gate drivers such that no additional supplies besides the positive and negative transmit voltage $( \pm 50 \mathrm{~V})$ and a control supply are required to power the drivers. Leakage currents through the high-voltage MOSFETs can significantly contribute to the heat generation as even small currents may cause a large dissipation due to the large current drop (100V). We chose this chip due to its fast $(<500 \mu \mathrm{s})$ power down mode reducing the MOSFET leakage below $10 \mu \mathrm{A}(<1 \mathrm{~mW})$.

Dedicated 8-channel TXBF chips (LM96570, TI) are used that create the pulser MOSFET control signals. The TXBF supports per-channel configurable bipolar pulse sequences with a frequency of 0.625 to $20 \mathrm{MHz}$ over a delay range of $102.4 \mu$ s with a resolution of 780 ps. Using TXBF chips does not allow completely arbitrary waveform generation, but simplifies the transmit path design compared to implementing the TXBF on the FPGA, which would require more I/O pins on the FPGA and more PCB routing space to connect all HV MOSFET switch signals to the FPGA. To support 64 channels, eight TXBF and pulser chips are placed on two 32-channel TX boards plugged to the motherboard from both sides.

The RX stage digitizes the received echoes. The ultrasound echoes cover a large dynamic range of over $100 \mathrm{~dB}$, due to the attenuation of the acoustic pulse as it is traveling through tissue. In practice, the full dynamic range is not needed all 
time and an ADC with lower dynamic range can be used in combination with a variable gain amplifier (VGA), which adjusts its amplification over time while capturing data. We selected an integrated analog front-end (AFE) chip (AFE5851, TI), which provides both a VGA (-5 to $31 \mathrm{~dB}$ ) and an ADC ( $12 \mathrm{~b}$ up to $32.5 \mathrm{MS} / \mathrm{s}$ ). The variable gain amplification profile is digitally programmed and its playback is triggered by the FPGA. The chip supports 16 channels at a very low power consumption of just $39 \mathrm{~mW}$ per channel. To achieve this power efficiency, two channels share a $65 \mathrm{MS} / \mathrm{s} \mathrm{ADC}$ in a timemultiplexed manner, which results in a half-sample period time-offset between the odd and even channels. This offset is compensated in the FPGA on the probe, such that the connecting system receives fully aligned samples. We selected this component due to its low active power consumption and because it supports two power down modes $(64 \mathrm{~mW}$ and $5 \mathrm{~mW}$ per chip), which can be can be left very quickly $(<50 \mu$ s and $<200 \mu \mathrm{s})$. Four AFE chips are used to provide 64 channels.

The AFE inputs are protected from the HV TX pulses with eight 8-channel T/R switches (TX810, TI) that clamp the input signal. The digital samples from the AFE are transferred to the FPGA (6.24 Gb/s per AFE chip) over a high-speed serial interface. All front-end chips are kept in sync with the required reference clocks and configured over a Serial Peripheral Interface (SPI) bus operating with up to $75 \mathrm{Mb} / \mathrm{s}$. A custom hardware SPI accelerator in the FPGA enables fast reconfiguration of the TX delays in the TXBFs between shots.

\section{FPGA Subsystem}

The FPGA aggregates the data from the ADCs and implements the high-speed link to the host system. A microprocessor instantiated on the FPGA runs the firmware to communicate with the host system and to control the probe.

The FPGA is provided on a compact $\left(4 \times 5 \mathrm{~cm}^{2}\right)$ commercial module (TE0712, Trenz Electronics). It features a Xilinx Artix-7 FPGA (XC7A200T-2FBG484C) with four $6.6 \mathrm{~Gb} / \mathrm{s}$ transceivers to connect the optical interface. The module has $8 \mathrm{~Gb}$ of DDR3 memory and $256 \mathrm{Mb}$ Quad-SPI Flash for configuration and operation. The chosen FPGA has plenty of spare resources to implement on-probe buffering or processing to connect as well with portable devices. We selected this module to speed up the implementation time of our probe and give us flexibility for future on-probe processing. Thus it is not particularly optimized for low power consumption.

The FPGA design (Fig. 4) contains a Xilinx MicroBlaze soft-core microcontroller with $256 \mathrm{kB}$ memory equipped with the required peripherals (timer, interrupt controller, I/O peripherals). A custom hardware finite-state-machine (LP FSM) is used to orchestrate the overall ultrasound acquisition depending on the settings requested by the connecting system. This state-machine allows precise PRF control and assures that the right number of samples are captured, and that the acquisition is in sync with the transmit pulse and the TCG profile.

The receive data-path on the FPGA from the AFE interface to the optical link contains three stages: First $\mathrm{AFE}$ Link abstracts the high-speed data-streams from the AFEs and interacts with the LP FSM to produce a data packet, which

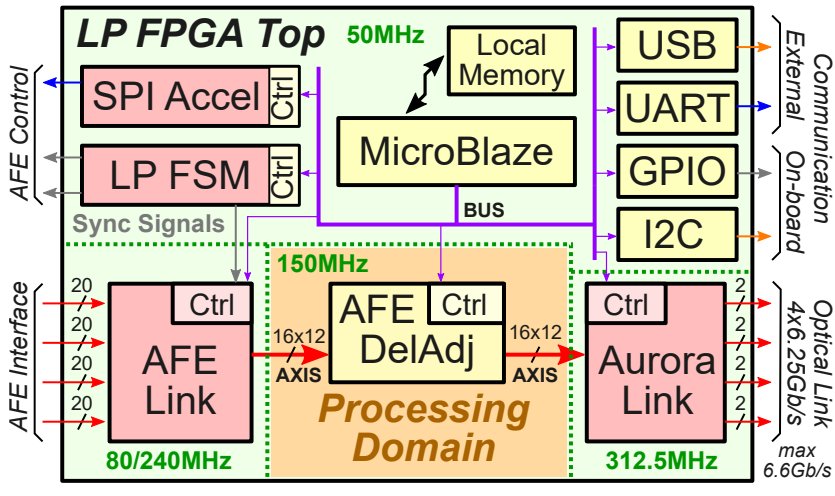

Fig. 4. LightProbe FPGA Top-level diagram: It features the control infrastructure (UPPER HALF) and the receive data-path (LOWER HALF). The control infrastructure is built around a soft-microcontroller (MicroBlaze) equipped with the required peripherals. A hardware finite-state-machine (LP FSM) orchestrates the overall signal acquisition by sending the required synchronization signals (TX-, TGC-, RX-trigger) with precise timing. The main data-path contains the AFE interface ( $\mathrm{AFE}$ Link), which produces per acquisition a raw data packet containing the samples of all channels. The data-packet may be processed in the processing domain on the probe, before it is forwarded to the optical interface block (Aurora Link).

contains the raw samples from all channels over a configurable time-period properly aligned to the transmit event. Second, the packet is forwarded to the processing domain, where any potential preprocessing block can easily be instantiated and connected. Even though we postulate and allow a complete software-defined system architecture, we still allow to perform hardware preprocessing in the FPGA on the probe. In the current design, we placed a delay adjustment (DelAdj) block that applies a $f_{s} / 2$ delay filter to the odd channels to compensate for the time-interleaved sampling of odd and even channels of our AFE chip. Additional processing blocks can be easily added. Third, the processed packet is forwarded to the Aurora Link which sends it to the PC using the Xilinx Aurora 8b/10b protocol taking care of DC-free coding and link synchronization. Due to the coding loss, only $80 \%$ of the link bandwidth is used for payload data. On the PC, the packet is received and its content is written into main memory using a direct-memory-access (DMA) transfer.

The maximal data rate of our optical link is $26.4 \mathrm{~Gb} / \mathrm{s}$ given by the maximal transducer speed of the FPGA transceivers $(4 \times$ $6.6 \mathrm{~Gb} / \mathrm{s})$. Given our $4 \mathrm{MHz}$ transducer we operate the ADCs with a $20 \mathrm{MS} / \mathrm{s}$ sampling rate producing a peak data rate of only $15.36 \mathrm{~Gb} / \mathrm{s}$. Considering coding loss and protocol overhead, the link is run at reduced speed $(6.25 \mathrm{GHz})$ providing a raw bandwidth of $25 \mathrm{~Gb} / \mathrm{s}$ over the four fibers in our cable.

The current design barely utilizes the FPGA (FF, LUT and DSP all $<30 \%$ ). Plenty of spare resources are available to extend the data-pipeline with additional processing.

\section{SyStem OpERATION AND FirmWARE}

The LightProbe provides a flexible model to program acquisition sequences (Fig. 5, LEFT). The basic building block of a sequence is a shot. A shot contains the transmit event, i.e. the emission of an ultrasound wave, followed by the receive phase, i.e. the period during which the echoes are captured. A $T X$ profile defines the parameters (delays, pulse shape) of 


\begin{tabular}{l|l|r}
$r^{T X_{1}} r^{T X_{2}} r^{T X_{3}}$ \\
$\mathrm{RX}_{\text {Shot }}^{\text {Burst }}$ & $\mathrm{RX}$ & $\mathrm{RX}$ \\
\hline $\mathrm{TX}$-config $\mathrm{t}$
\end{tabular}

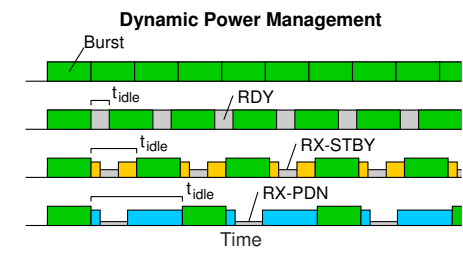

Fig. 5. Acquisition Sequence Programming (LEFT): A burst contains multiple shots. A shot is a TX event followed by a RX phase. Dynamic power management (RIGHT): If the idle period $\left(t_{\text {idle }}\right)$ between two bursts is long enough for a power state transition (yellow, blue), the corresponding power mode is activated to reduce the power dissipation during the idle period.

the emitted wave. Multiple shots can be combined to a burst. Within a burst, every shot can use a different TX profile.

Since changing the TX profile requires to reconfigure the TXBF chips, a brief re-configuration phase is inserted. Depending on the triggering settings, shots, burst or multiple bursts can be launched with a single trigger command issued by the ultrasound application running on the host PC. Upon receiving a trigger signal, LIGHTPROBE emits the ultrasonic pulses, captures the echoes and sends the data to the host.

\section{A. Dynamic Power Management}

As elaborated in Sec. II, fast and good power down modes are required to benefit from the power savings possible by imaging strategies that allow duty-cycling of the front-end.

To keep the probe as cool as possible all unneeded modules must be prevented from generating heat. In a complex system as the LightPROBE, where individual supplies can be turned off and many chips support many different power modes, the size of the combined state space of feasible system power states explodes. We selected the most useful in terms of transition time and the power savings they provide:

ready (RDY): the entire front-end is powered up, the RX path is sampling and the TX path is ready to emit pulses. The probe has to be in this state to acquire raw data.

power down (PDN): the front-end power supplies are shut down. Recover operation from this mode takes $350 \mathrm{~ms}$ due to the time required to recharge the supply domains. receive power down ( $\mathrm{RX}-\mathrm{PDN})$ : the power supplies remain on, but the TR switches, the TX pulsers and the AFE are put in power down, which also disables the AFEFPGA link. Recovering from this modes requires to resynchronize the AFE-FPGA links, which takes $25 \mathrm{~ms}$.

receive standby ( $\mathrm{RX}-\mathrm{STB} \mathrm{Y}$ ) the TR switches, the TX pulsers as well as the ADCs are in standby. The AFE-FPGA link is only partially powered down to keep the synchronization, which enables to resume operation in only $600 \mu \mathrm{s}$.

The dynamic power management, which is part of the firmware, automatically activates the power down state with the highest possible power savings to keep the average power consumption as low as possible (Fig. 5, RIGHT). To do this, it first computes the length of the idle period $\left(t_{\text {idle }}\right)$ between two bursts from the current imaging settings (FPS, PRF, RX period) and then selects the best possible power mode to enter given its transition time and overhead. The selected mode is activated immediately after the last receive phase of the burst. A timer is set to schedule the wakeup, such that the probe will be back in RDY shortly before the next shot starts.

\section{B. Dynamic Thermal Management}

Digital ultrasound probes, like other devices embedding active electronics, are thermally limited systems, which means for some operating modes they can produce more heat than can be dissipated. Continued operation within these operating modes can cause the device to heat up to a point where it exceeds prescribed thermal limits for safe operation.

Some ultrasound probes use active cooling. Air cooling is not a viable option, since air inlets and moving rotors would impede proper disinfection of the probe. One solution is to use fluid cooling through the cable, which has been implemented in probes for 3D imaging [29]. Passive cooling is the only option to keep complexity and cost within reasonable bounds.

Relying on passive cooling requires the average dissipated power to stay below a certain level to assure that the surface temperature never exceeds $43^{\circ} \mathrm{C}$ to comply with medical safety regulations (IEC 60601-1). One way to ensure this, it to restrict the probe to modes, which when operated continuously in worst-case conditions (high ambient temperature) never heat the probe beyond $43^{\circ} \mathrm{C}$. This puts overly strict restrictions on the system, as it is seldom in worst-case conditions and some modes do not need to be operated continuously. Another approach is to adapt the operation mode (or its parameter) depending on the current temperature of the probe.

The firmware of LIGHTPROBE features a dynamic thermal management to enable this kind of thermal aware operation: The probe is equipped with multiple temperature sensors that are used as an input for our thermal control algorithms. As highlighted in Fig. 3 temperature sensors are placed at critical locations close to all major heat sources, which are the FPGA, the optical transceiver, the AFE chips, the TX pulsers and the HV supplies. For each temperature sensor we define a maximal tolerable value $T_{\max , i}$, such that when reached, the outside surface temperature does not exceed regulatory limits. We define the thermal margin $T_{\mathrm{m}}$ as the minimal margin over all sensors. The firmware computes $T_{\max , i}$ every second:

$$
T_{\mathrm{m}}=\min _{i} T_{\max , i}-T_{i}
$$

The milled aluminum housing of the LightProbe (Fig. 3) features thermal contact areas to the FPGA and PCB stack to ease heat spreading, and provides sufficient heat capacity to temporarily sink the heat during high-performance modes without increasing the surface temperature significantly.

In this work, we implemented two thermal controllers:

- First, a Thermal-Aware-Performance (TAP) controller, that adapts imaging settings (the frame-rate in this work) to keep the probe below a given temperature.

- Second, a Boost-Mode (BM) controller that allows dualmode operation of a continuously operable default mode (B-mode) and a boost mode ( 2 seconds of $210 \mathrm{~Hz}$ imaging) with a power consumption exceeding the sustainable cooling capabilities. The boost mode can be activated ondemand with a defined periodicity (every 5 seconds) to provide a consistent Quality of Service (QoS).

The first controller (TAP) sets the frame-rate depending on $T_{\mathrm{m}}$ by inserting a delay $d_{\mathrm{th}}\left(T_{\mathrm{m}}\right)$ between the triggering of the bursts. The dynamic power management (Sec. V-A) 


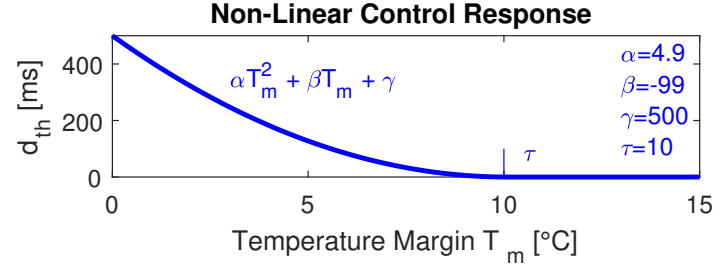

Fig. 6. Non-linear control response used by the TAP controller.

takes care of activating the best power down mode between bursts. Designing the controller response $d_{\mathrm{th}}\left(T_{\mathrm{m}}\right)$ is not straight forward as the controlled variable, i.e. the temperature, reacts with a delay to control inputs, i.e. frame-rate changes. Controlling a system with a delayed response using a standard $\mathrm{P}(\mathrm{ID})$-controller is susceptible to instability. Instability can be avoided by setting the slope of the linear control response very flat. However, the slope cannot be set arbitrarily flat as at a certain temperature, a specific frame-rate is required to avoid the system from overheating. In our case, controller and thermal stability was not achievable with a linear control response. We thus designed $d_{\mathrm{th}}\left(T_{\mathrm{m}}\right)$ as a one-sided quadratic function (Fig. 6), which has a low response when sufficient thermal margin is available (stability) and a progressive response on decreasing thermal margin (overheating avoidance).

$$
d_{\mathrm{th}}\left(T_{\mathrm{m}}\right)= \begin{cases}\alpha T_{\mathrm{m}}^{2}+\beta T_{\mathrm{m}}+\gamma, & \text { if } T_{\mathrm{m}} \leq \tau \\ 0, & \text { otherwise }\end{cases}
$$

If the probe is cool enough $\left(T_{\mathrm{m}}>\tau=10^{\circ} \mathrm{C}\right)$, the thermal management does not interfere and the probe runs at the configured frame-rate. For smooth frame-rate changes and to remove sensor noise, a first-order IIR low-pass filter is applied to $d_{\text {th }}$. Currently, TAP only sets the frame-rate. Other settings could be controlled as well. We use this controller to provide normal B-mode imaging at best possible performance (framerate) in all temperature conditions.

The second controller (BM) uses a TAP controller with a modified set-point ( $\tau$ set to $15^{\circ} \mathrm{C}$ ) to let the probe cool down during default mode in order to build up additional thermal margin for the boost mode. A LED informs the user when the system is thermally ready $\left(T_{\mathrm{m}}>T_{\mathrm{m} \text {, bst rdy }}=10^{\circ} \mathrm{C}\right)$ to switch to the boost mode. The user can do so by pressing a button on the probe. By choosing the set-point of the default mode (using $\tau$ ) and the thermal margin for activation $\left(T_{\mathrm{m} \text {, bst rdy }}\right)$ properly depending on the heat output of the boost mode, it can be ensured that the boost mode can be activated with a lower bounded duty cycle, without ever overheating the system.

\section{METHODS}

\section{A. Imaging Quality Assessment (Resolution, Contrast, Noise)}

Fig. 7 depicts the overall test system. For details about the PC system see [25]. In order to assess the imaging quality of the LightProbe, we implemented several imaging strategies (both conventional and ultrafast) and quantified the achieved contrast and resolution. For the resolution and contrast measurements a CIRS 054GS phantom $(0.5 \mathrm{~dB} / \mathrm{cm}-\mathrm{MHz})$ is used. If not stated otherwise a $4 \mathrm{MHz} 2$ period $[+1,-1,+1,-1]$ transmit pulse is used. All channel were sampled with a sampling frequency of $20 \mathrm{MHz}$ and the RF data was processed offline.

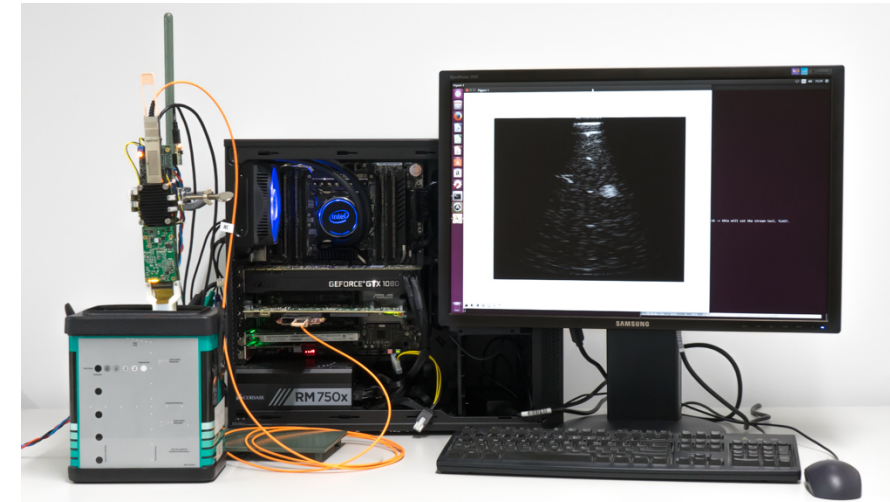

Fig. 7. Test system of the LightPROBE in operation connected to the PC. See [25] for more details about the test system.

For all strategies, the following processing steps were performed: The raw RF data was bandpass filtered and converted into analytical signals. Dynamic receive beamforming was performed on a polar grid with a dynamic hanning apodization. After beamforming we performed 2D scanline interpolation, envelop extraction and scan-conversion. The quality metrics were extracted and the images were log-compressed for displaying. The following imaging strategies were evaluated:

TXF1 Conventional line-by-line imaging with dynamic receive focusing on 91 scanlines $\left(0.86^{\circ}\right.$ spacing, $78^{\circ}$ fieldof-view) and a fixed transmit focus at $5 \mathrm{~cm}$.

TXF2 Same as TXF1 but with sequential multi-zone transmit focusing to 5 and $10 \mathrm{~cm}$. The scanlines from the two acquisitions with different transmit focus were linearly blended along the depth.

STA64 synthetic transmit aperture imaging [30] with 64 sequential single element isonifications. 64 low-resolution images were coherently summed to one high-resolution image (HRS) before envelop extraction.

PW31 coherent-compounded plane-wave imaging using 31 waves with angles from $-15^{\circ}$ to $+15^{\circ}$ with $1^{\circ}$ increments.

VS13 synthetic aperture imaging with 13 virtual sources [31], [32] placed behind the transducer on a $90^{\circ} 6.3 \mathrm{~mm}$ radius arc. The 13 LRS images were coherently combined.

VS13-CE same as VS13, but extended with coded excitation [33], [34] using two 48 bipolar pulse sequences emitted with $8.88 \mathrm{MHz}$. The two acquisitions were decoded and combined before fed to the normal processing pipeline.

For every strategy we quantify the resolution at different depths with the full-width at half-maximum (FWHM) of the vertical point targets in the phantom. The theoretical FWHM in degrees of the system assuming full use of the aperture for transmit and receive is according to [35] $\phi_{\mathrm{th}}=1.206 \mathrm{\lambda} / \mathrm{a}$. $360 / 2 \pi=2.11^{\circ}$ with $a$ the transducer aperture size $(1.26 \mathrm{~cm})$ and $\lambda$ the wavelength $(0.385 \mathrm{~mm})$. Contrast is quantified using the anechoic cylinders in the phantom using the contrast ratio (CR) [35] and the contrast-to-noise-ratio (CNR) [36]:

$$
\mathrm{CR}=\frac{\mu_{\text {out }}-\mu_{\text {in }}}{\mu_{\text {out }}+\mu_{\text {in }}}, \quad \mathrm{CNR}_{\mathrm{dB}}=20 \log _{10} \frac{\mu_{\text {in }}-\mu_{\text {out }}}{\sqrt{\left(\sigma_{\text {in }}^{2}+\sigma_{\text {out }}^{2}\right) / 2}}
$$

with $\mu$ the mean and $\sigma^{2}$ the variance of the B-mode image within and around the cyst. The signal-to-noise ratio $(S N R)$ as 
a function of depth is computed along the center line in the BMode picture before log-compression using mean and standard deviation values obtained from 100 consecutively captured images of a CIRS 040GSE phantom using VS13:

$$
\operatorname{SNR}_{\mathrm{dB}}(z)=20 \log _{10}(\mu(z) / \sigma(z)) .
$$

\section{B. PRF, Frame-Rate and Power Measurements}

The maximal achievable pulse repetition frequency (PRF) is physically limited by the time it takes the acoustic pulse to reach the desired imaging depth and for the echoes to return back to the transducer. In a real system, the achievable PRF is further limited by configuration overheads between acquisitions and the data-sinking capabilities of the receive path. Since the digital link of LiGHTPROBE allows to continuously stream the received data off the probe, we can operate the system perpetually with a very high PRF as opposed to systems, which have to periodically stall their operation to deplete internal buffers over a low-rate link.

For the power and PRF measurements, we configured the probe to take 2048 samples per shot, which takes $102.4 \mu \mathrm{s}$ with a sampling rate of $20 \mathrm{MHz}$. Thus, a maximum PRF of $9.8 \mathrm{kHz}$ can be reached in theory. To measure the consumed power, the probe was supplied with a Keysight N6705B DC Power Analyzer instead of using the USB port of the PC.

\section{Thermal Control}

Both thermal-aware controllers where implemented and executed on the LightPROBE, while the power consumption and achieved frame-rate was captured. Profiling starts from a cool system $\left(21^{\circ} \mathrm{C}\right.$ ambient temperature). The probe was configured for the PW31 imaging scheme with the 31 acquisitions performed in $6.5 \mathrm{kHz}$ PRF burst. To obtain more conservative results, we chose PW31 over VS13 as it requires more shots per frame compared to the powered-optimized VS13 operating with 13 shots only. For normal B-mode imaging, the freerunning frame-rate is set to $210 \mathrm{~Hz}$. The thermal controller will reduce the frame-rate to a steady-state value that is thermally sustainable. This high free-running frame-rate is chosen to force reasonably fast convergence to the steady-state value. The boost-mode is configured as a 2 second period of acquiring PW31 frames at a rate of $210 \mathrm{~Hz}$. This setting is reasonable to capture a flow sequence, which can be replayed at lower frame-rate. Controller input and output variables are extracted over a debug interface.

\section{RESULTS}

\section{A. Imaging Quality Assessment}

Fig. 9 shows the imaging output for all strategies. We show a wide-angle image of the CIRS phantom down to $9 \mathrm{~cm}$ with the vertical point targets aligned on the center axis of the transducer. The lateral (axial) resolution is quantified for every point target by measuring the FWHM in degrees. We plot the depth resolution profile and report the average resolution over the depth $2-9 \mathrm{~cm}$.

The system resolution is almost perfectly achieved for STA64 and PW31 over the entire depth, which is expected for these strategies. For TXF1 and TXF2 this is only the case at their transmit focal depths (5 and $10 \mathrm{~cm}$ ), which is expected as well. VS13 achieves a slightly worse resolution, but also needs by far the least amount of shots to obtain an image. The codedexcitation in VS13 impairs the resolution only marginally.

For contrast, we show the image section around the anechoic cylinder at $4 \mathrm{~cm}$ depth and report the CR and CNR using the regions indicated in the image. As expected STA64 provides a poor contrast (CNR: $3.42 \mathrm{~dB}$ ) due to the little amount of acoustic energy emitted into the tissue by using only a single transducer element for transmission. Using all elements for transmit yields a better CNR for the other synthetic aperture based methods PW31 and VS13 (CNR: $6.86 \mathrm{~dB}$ and $7.03 \mathrm{~dB}$ ). As expected, the best contrast is achieved with conventional sequential scanning (TXF1, TXF2) which uses transmit focusing (CNR: $7.60 \mathrm{~dB}$ and $7.58 \mathrm{~dB}$ ). Coded excitation boosts the penetration depth and increases the VS13 contrast at $4 \mathrm{~cm}$ depth from $7.03 \mathrm{~dB}$ to $7.17 \mathrm{~dB}$ and at depth $7 \mathrm{~cm}$ from $3.56 \mathrm{~dB}$ to $6.63 \mathrm{~dB}$ (see Fig. 8), but requires twice the number of shots and has a more energy intensive TX pulse.

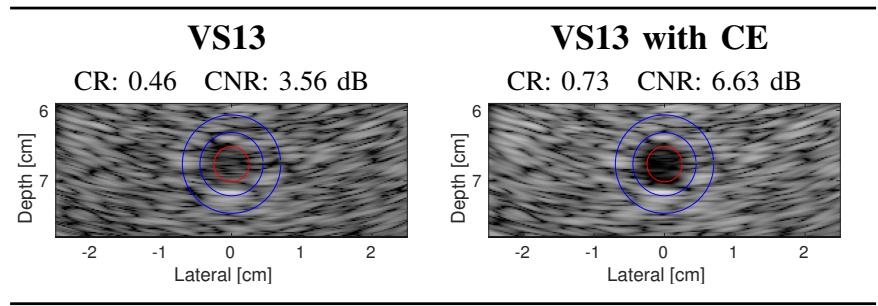

Fig. 8. Boosting the penetration depth with coded excitation

This evaluation confirms that with ultrafast methods (PW31 \& VS13) almost the same imaging quality can be achieved for still images as with standard methods. Compared to TXF2, the ultrafast methods PW31 and VS13 require $6 \times$ and $14 \times$ less shots, while dropping the average resolution less than $0.3^{\circ}$ (VS13) and the contrast less than $0.72 \mathrm{~dB}$ (PW31).

\section{B. Achievable PRF and Frame-Rate}

In the standard case, where the TX profile needs to be updated between the shots, all delays $(1.8 \mathrm{~kb})$ are programmed into the TXBF chips in $25 \mu$ s with our SPI-HW-accelerator. In this case, the LIGHTPROBE achieves a PRF of $6.5 \mathrm{kHz}$.

The maximal frame-rates we can achieve for the various imaging strategies with a $6.5 \mathrm{kHz}$ PRF are listed in Tbl. II. With all strategies we reach a frame-rate above $30 \mathrm{~Hz}$. With VS13, we can achieve $500 \mathrm{~Hz}$. The high-frame-rate operation is demonstrated with videos in the supplementary material ${ }^{1}$. In a follow-up work [37], we use the LIGHTPROBE to track trajectories of individual gas bubbles at $200 \mathrm{fps}$.

For these very high frame-rates, we buffer the raw data on the PC's main memory and interrupt the acquisitions after a few seconds to store the data on disk to processes it later offline. Note that these high frame-rates are sustainable by state-of-the-art GPU-based beamformers [2], [3].

${ }^{1}$ http://hdl.handle.net/20.500.11850/283465 

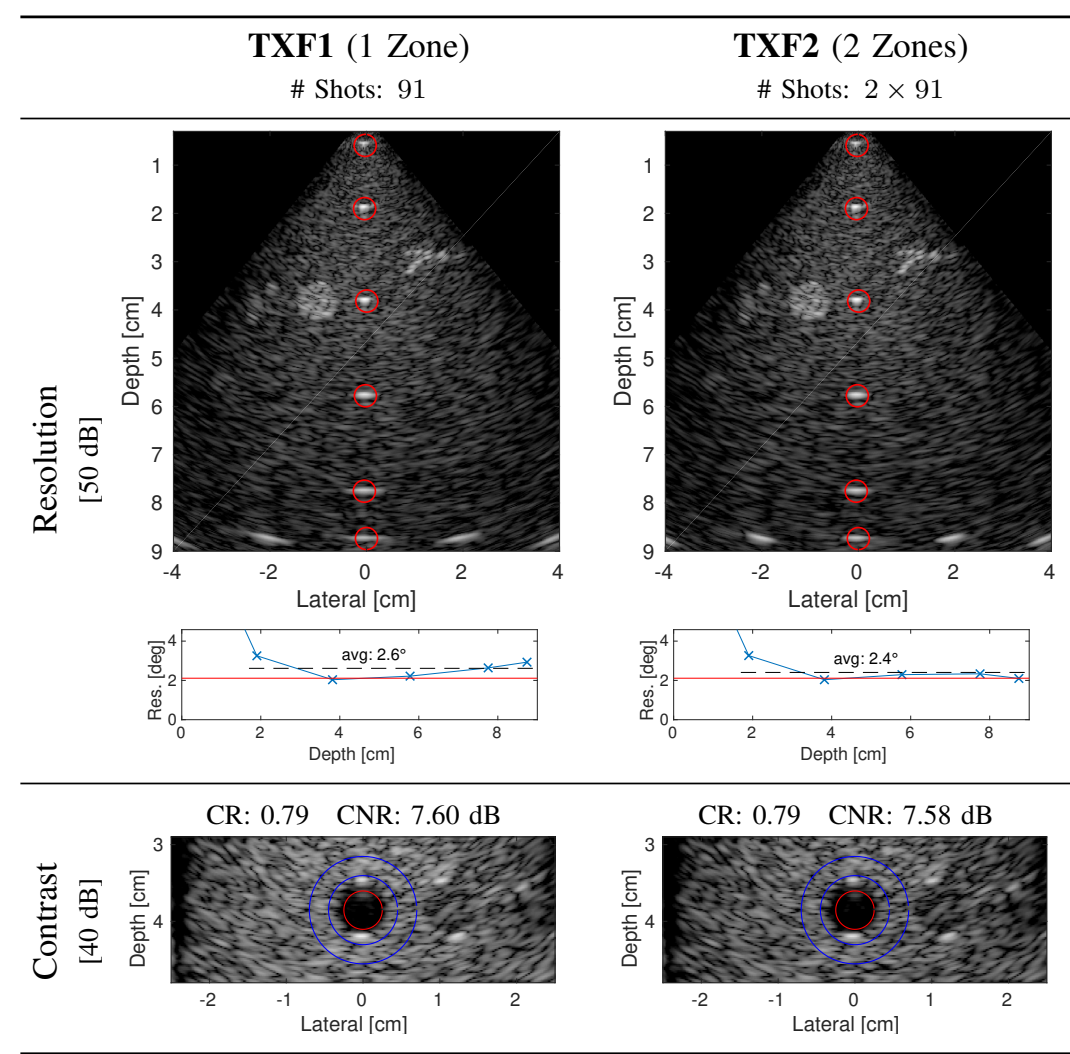

STA64
\# Shots: 64

\begin{tabular}{ccc}
\hline \hline PW31 & VS13 & VS13 with CE \\
\# Shots: 31 & \# Shots: 13 & \# Shots: $2 \times 13$ \\
\hline
\end{tabular}
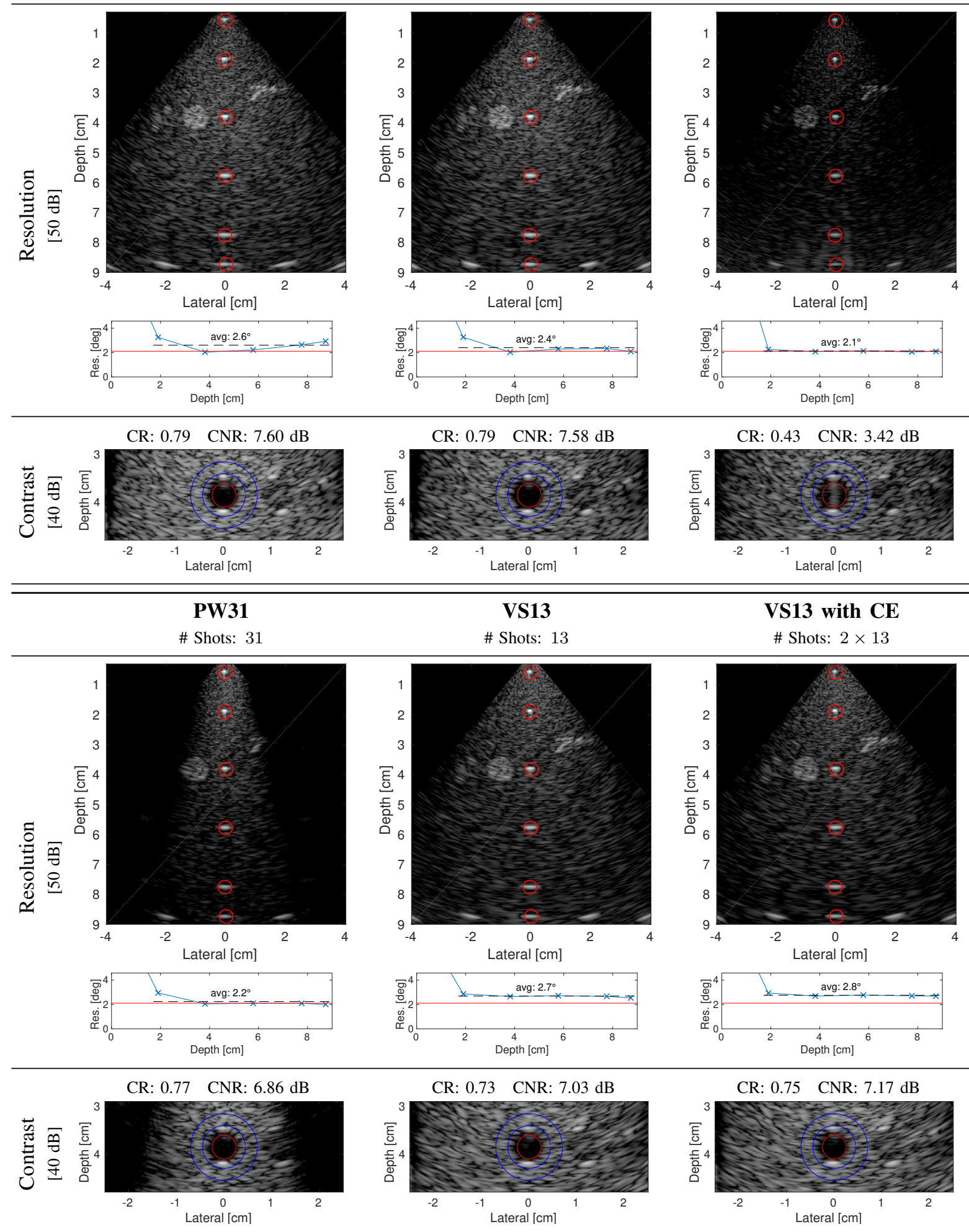

Fig. 9. Imaging quality of the LightPROBE for different transmit strategies: For each strategy, the lateral (axial) $-6 \mathrm{~dB}$ point spread is measured at multiple depths (red circles), as well as the contrast [CR] and contrast-to-noise [CNR] ratio of an anechoic region. For the lateral resolution we report the average angle spread for the points at 2-9 $\mathrm{cm}$ depth. The resolution profile shows the theoretical system FWHM resolution (red line) in degrees $\phi_{\text {th }}$. For each strategy the number of required acquisition per frame (shots) is listed. The resolution images are shown with $50 \mathrm{~dB}$ dynamic range and the contrast images with $40 \mathrm{~dB}$. 


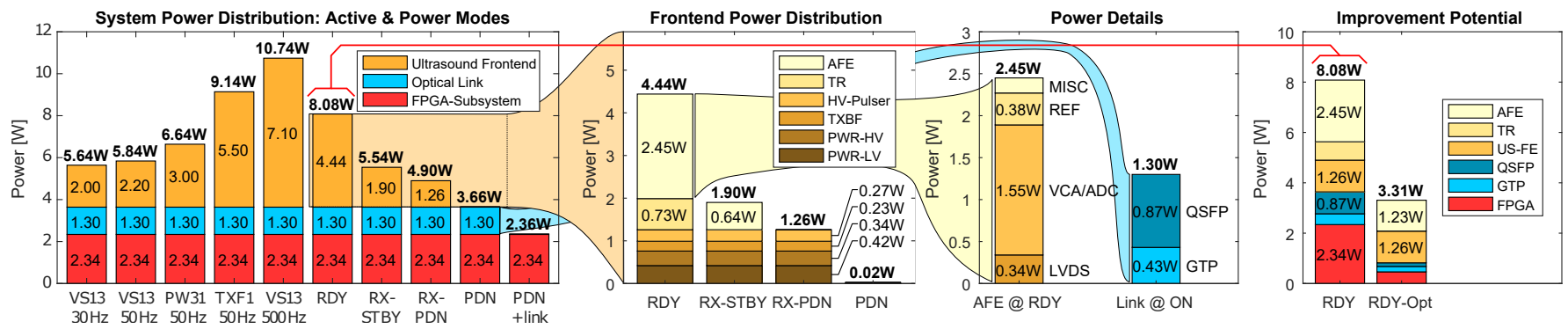

Fig. 10. LightProbe Power Measurements: Left: LightProbe power consumption (measured) in various operation mode and power states (see text), showing the power contributions from the Ultrasound front-end, Optical Link (including transceiver in FPGA) and FPGA-Subsystem. MIDDLE LEFT: More detailed power analysis of the ultrasound front-end showing the power distribution in the four main power modes. MIDDLE RIGHT: Detailed power breakup of the AFE in RDY and the optical link when on. Top RIGHT: Potential power improvements for RDY (estimated), see Section VIII for details.

\section{Power Measurements}

Fig. 10 shows a detailed power breakup of the LIGHTPROBE for different power state and operation modes: If the probe is in RDY state $(8.1 \mathrm{~W})$ and not emitting pulses, more than half of the power $(4.4 \mathrm{~W})$ is spent in the ultrasound frontend. The largest contribution to the front-end power is the AFE $(2.45 \mathrm{~W})$. Thus the AFE should be turned off whenever possible. If the PRF and frame-rate settings allow, the frontend can enter the RX-STBY or RX-PDN power mode in between acquisitions consuming only $1.9 \mathrm{~W}$ or $1.26 \mathrm{~W}$. In idle periods $>350 \mathrm{~ms}$, the front-end can be powered down (PDN) consuming as little as $25 \mathrm{~mW}$. If the probe is performing highframe-rate ultrafast imaging (VS13, $500 \mathrm{~Hz})$ the probe power consumption rises to over $10 \mathrm{~W}$ due to the additional power spent for emitting pulses. If the probe is performing normalrate $\mathrm{B}$-mode imaging $(\mathrm{VS} 13,30 \mathrm{~Hz})$ the power consumption decreases below the idle level (RDY) as more power is saved with power management than used for transmission.

We report the front-end power consumption separately from the FPGA subsystem and the optical link. Note that we focused in this work on reducing the consumption of the front-end as it cannot be scaled down as easily as the digital counterparts. Currently, $3.64 \mathrm{~W}$ are spent for the FPGA subsystem and the optical link independent of the operating mode. As elaborated in Sec. VIII these parts can be further optimized.

TABLE II

Ultrasound FRont-end Power WITH Dynamic Power Management

\begin{tabular}{llrrr}
\hline MODE & $\begin{array}{l}\text { max. FPS } \\
(6.5 \mathrm{kHz})\end{array}$ & $\begin{array}{r}\text { Avg Pwr } \\
\text { max. FPS }\end{array}$ & $\begin{array}{r}\text { Avg Pwr } \\
50 \mathrm{~Hz}\end{array}$ & $\begin{array}{r}\text { Avg Pwr } \\
30 \mathrm{~Hz}\end{array}$ \\
\hline TXF2 & $36 \mathrm{~Hz}$ & $7.1 \mathrm{~W}$ & $\mathrm{n} / \mathrm{a}$ & $6.3 \mathrm{~W}$ \\
TXF1 & $71 \mathrm{~Hz}$ & $7.1 \mathrm{~W}$ & $5.5 \mathrm{~W}$ & $3.9 \mathrm{~W}$ \\
PW31 & $210 \mathrm{~Hz}$ & $7.1 \mathrm{~W}$ & $3.0 \mathrm{~W}$ & $2.4 \mathrm{~W}$ \\
VS13 & $500 \mathrm{~Hz}$ & $7.1 \mathrm{~W}$ & $2.2 \mathrm{~W}$ & $2.0 \mathrm{~W}$ \\
\hline
\end{tabular}

Tbl. II reports the ultrasound front-end power consumption for various imaging modes (TXF2, TXF1, PW31, VS13) running at their maximal possible frame-rate as well as rates for B-mode imaging $(30$ and $50 \mathrm{~Hz}$ ). Even though the modes provide substantially different maximal frame-rates $(36-500 \mathrm{~Hz})$, all modes consume around $7.1 \mathrm{~W}$ at their max-rate, as raw data is acquired with the same $6.5 \mathrm{kHz}$ PRF. VS13 provides a $14 \times$ higher frame-rate compared to TXF2 at the same power consumption.

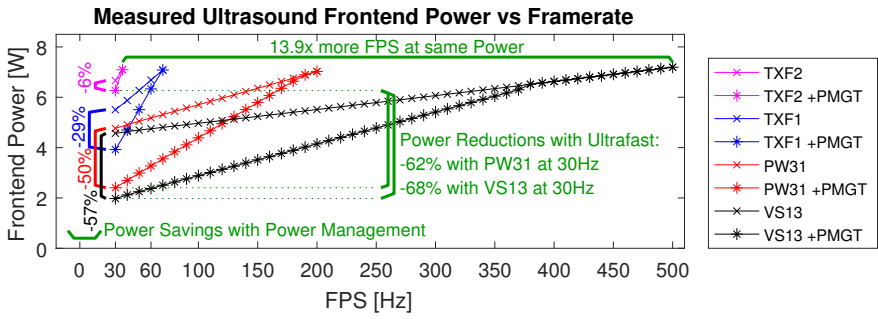

Fig. 11. Measured ultrasound front-end power consumption for different imaging strategies (TXF2, TXF1, PW31, VS13) and frame-rates $(30 \mathrm{~Hz}$ to max) with and without dynamic power management active.

Fig. 11 shows the measured ultrasound front-end power during imaging for various modes and frame-rates $(30 \mathrm{~Hz}$ to $\max$ ), with and without dynamic power management activated. If it is deactivated and no power down modes are utilized, the front-end power consists of a static contribution (RDY power, $4.4 \mathrm{~W}$ ) and a contribution growing proportionally with the frame-rate accounting for the power dissipated for ultrasound pulse emissions (TX). With dynamic power management the average power consumption can be substantially reduced when there is sufficient idle time $\left(t_{\text {idle }}\right)$ between frames to put the front-end in to a power-down mode. At $30 \mathrm{~Hz}$ power savings of $-6 \%$ (TXF2), -29\% (TXF1), -50\% (PW31) and -57\% (VS13) can be achieved. For normal B-mode imaging a frame-rate of $30 \mathrm{~Hz}$ is sufficient. If ultrafast imaging (PW31, VS13) is used at this low rate, the average power consumption is drastically reduced (-62\% $2.4 \mathrm{~W}$ PW31, $-68 \% 2.0 \mathrm{~W}$ VS13) compared to conventional imaging (TXF2, $6.3 \mathrm{~W}$ ) as the frontend can be powered down longer between frames. Note that without dynamic power management, the savings with ultrafast imaging would be less than half, only $-29 \%$ in PW31 and $32 \%$ in VS13. This shows that dynamic power management is crucial to realize the potential savings ultrafast method offer.

\section{Thermal Control}

Fig. 13 shows the TAP controller performance starting from a cold system $\left(21^{\circ} \mathrm{C}\right)$ until the system reaches a steadystate. At first, the system is running with the configured freerunning frame-rate $(210 \mathrm{~Hz})$ for $3 \mathrm{~min}$, heating up until the thermal margin $\left(T_{\mathrm{m}}\right)$ is reduced to $10^{\circ} \mathrm{C}$ and the controller throttles the systems frame-rate by adding a delay $\left(d_{\mathrm{th}}\right)$ in between capturing frames. Immediately after throttling starts, 


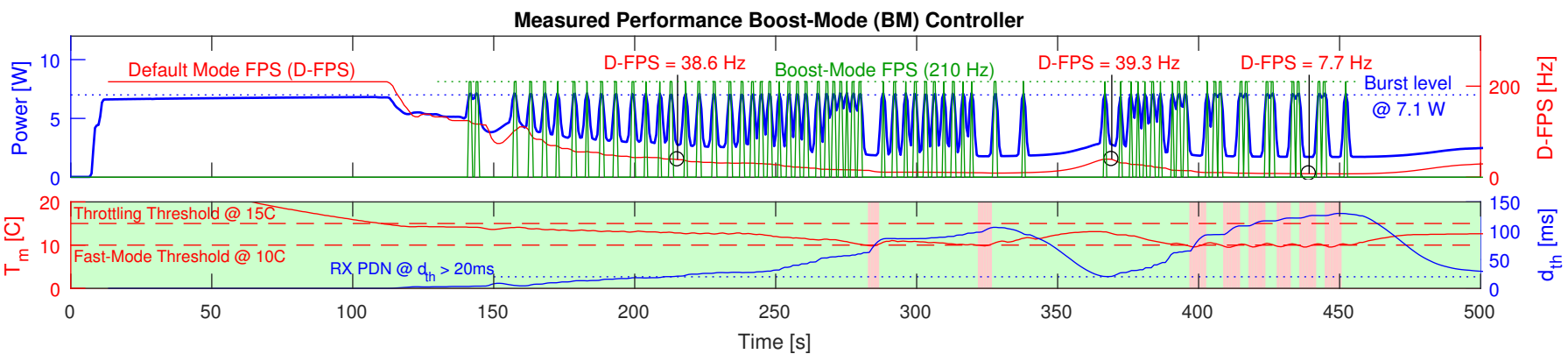

Fig. 12. Measured performance of the LightProbe using the Boost-Mode (BM) controller: The top plots shows again the average power consumption of the front-end (blue) and the lower plot shots the thermal margin $T_{\mathrm{m}}$ in ${ }^{\circ} \mathrm{C}$ (red) and the manipulated variable $d_{\mathrm{th}}$ of the controller (blue). Initially, the system operates in the default imaging mode with a thermally-adapted frame-rate (D-FPS, red) and a boost mode (green) with a FPS of $210 \mathrm{~Hz}$ (PRF $6.5 \mathrm{kHz}$ ) for $2 \mathrm{~s}$. The high performance mode can be temporarily activated upon user request when $T_{\mathrm{m}}>T_{\mathrm{m}}$, bst rdy $=10^{\circ} \mathrm{C}$. The background color of the bottom plot indicates whether the mode can be activated (green) or not (red).

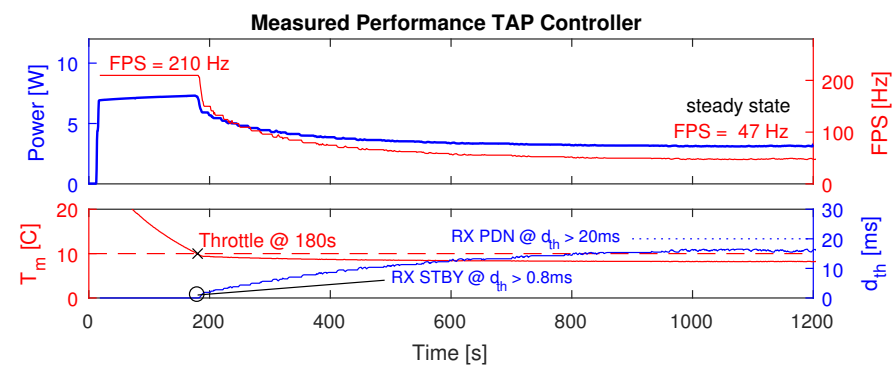

Fig. 13. Measured performance of the LIGHTPROBE using the ThermalAware-Performance (TAP) controller: The top plots shows the average power consumption of the front-end (blue) along with the instantaneous frame-rate (FPS, red). The lower plot shots the thermal margin $T_{\mathrm{m}}$ in ${ }^{\circ} \mathrm{C}$ (red) and the manipulated variable $d_{\text {th }}$ of the controller (blue). Controlling the frame-rate in combination with activating the provided power down mode allows the system to reach a steady state, which still provides a frame-rate of $47 \mathrm{~Hz}$.

the added delay $d_{\text {th }}$ is long enough ( $>0.8 \mathrm{~ms}$ ) to activate RXSTBY between frames, which reduces the average power consumption. The system continues to slowly heat up for the next 15 minutes until it reaches a steady state with $T_{\mathrm{m}}>5^{\circ} \mathrm{C}$ and a frame-rate of $47 \mathrm{~Hz}(1.5 \mathrm{kHz}$ PRF in average). Currently, $T_{\max , i}$ is set to $60^{\circ} \mathrm{C}$ and $70^{\circ} \mathrm{C}$ for the PCB and FPGA sensors respectively. With these values, the $\mathrm{PCB}$ temperature reaches around $52^{\circ} \mathrm{C}$, which keeps the housing surface below $43^{\circ} \mathrm{C}$ under standard ambient temperatures $\left(21^{\circ} \mathrm{C}\right)$. If the system would further heat up, e.g., when a more power intensive transmit pulse is used, $d_{\text {th }}$ would be further increased until at $20 \mathrm{~ms}$ there is enough time to activate RX-PDN.

Fig. 14 (LEFT) shows a thermal camera (FLUKE Ti95) image of the housing of the probe showing that the outside temperature stays below $43^{\circ} \mathrm{C}$. Note that the transducer tip is currently the hottest part of the probe. This is only the case if the tip is not touching any surface (skin or phantom) serving as a cooling path. Note that the transducer temperature can be easily reduced by providing a heat conductance path to the metal housing, which is currently not given as the connecting parts (Fig. 3) are made out of ABS plastic.

Fig. 14 (RIGHT) shows the SNR of a B-mode image (VS13) along the center-line as a function of depth at different internal probe temperatures at the AFE chips. Over our temperature range $\left(30-50^{\circ} \mathrm{C}\right)$ the noise performance is constant.

While simple controllers, such as the TAP, successfully
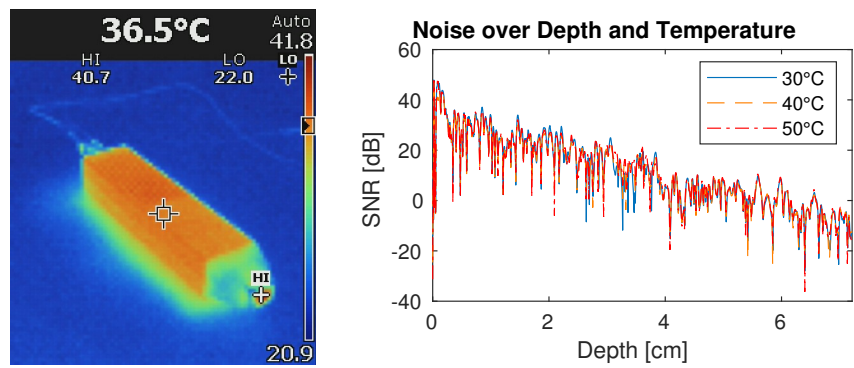

Fig. 14. Thermal Performance: LEFT: Thermal camera picture of the LightProbe in thermal regulation. The housing temperature is $<37^{\circ} \mathrm{C}$ and the transducer $41^{\circ} \mathrm{C}$, both are not exceeding $43^{\circ} \mathrm{C}$. Right: Noise measurement depending on depth and temperature. Note: SNR measurement should not be compared with Fig.9 as a phantom with stronger attenuation $(0.7 \mathrm{~dB} / \mathrm{cm}-\mathrm{MHz})$ was used for the noise measurement.

avoid overheating, they do not provide a consistent QoS, since after providing the peak performance for a few minutes, they throttle the system and stay in throttled mode indefinitely, unless the probe is turned off for cooling.

Fig. 12 shows the system performance using the boost-mode (BM) controller, which supports dual-mode imaging with a frame-rate controlled default mode (D-Mode) and a BoostMode that can be activated on-demand. After start-up the DMode provides again peak performance for a few minutes (20-100s) before it is throttled. Activating the Boost-Mode at a high rate (150-300s in Fig. 12) accelerates the heating process. Note that now the frame-rate of D-Mode (D-FPS) drops sufficiently low $\left(d_{\mathrm{th}}>20 \mathrm{~ms}\right)$ to activate the second power down mode RX-PDN in between frames. At one point $T_{\mathrm{m}}$ reaches $T_{\mathrm{m} \text {, bst rdy }}=10^{\circ} \mathrm{C}$ (@280s) and the user is no longer allowed to activate Boost-Mode for a few seconds until the system has cooled a bit. When Boost-Mode is not activated for a while, the system cools and D-FPS is increased again (@ 350s). We demonstrate a consistent Boost-Mode launch rate (once every 5 seconds) by keeping the activation button pressed (400-450s). During these 50 s 11 Boost-Mode bursts are launched. Even now, D-Mode still provides a framerate $>6 \mathrm{~Hz}$. With the $\mathrm{BM}$ controller we demonstrate that we can ensure the temporary use of a high-performance mode dissipating more energy than what is thermally sustainable in continuous operation, while still providing a consistent QoS for the operator. 


\section{DISCUSSION AND COMPARISON}

Our power results in Tbl. II confirm that digital probes can significantly reduce (more than $-60 \%$ ) the average front-end power consumption during normal rate $(30 \mathrm{~Hz}) \mathrm{B}$-mode imaging by implementing ultrafast imaging methods in combination with duty-cycling of the front-end. In all normal-rate B-mode scenarios (VS13, PW31: 30-50 Hz), we are able to apply dutycycling to reduce the overall front-end power consumption to even lower values (2.0-3.0 W) than the idle consumption of an always-on front-end (RDY, $4.4 \mathrm{~W}$ ). This is a very fundamental observation as digital probes with many channels $(>32)$ are often regarded unfeasible as an always-on front-end is assumed and the consumption of the ADCs is simply added up, without considering the additional power saving potential that using more ADCs to support ultrafast imaging can provide.

In Tbl. III we compare the LIGHTPROBE with both systems and concepts reported in the literature as well as commercial systems. With the LightProbE we can support the same imaging modalities (B/M-Mode, Color Doppler) as current digital probes for portable imaging do [16]-[18], [38]-[41]. On top of this, we support modalities that are typical of highend systems, which we demonstrated so far with the highspeed imaging example. LIGHTPROBE provides real-time raw sample access. This feature is currently only supported by topof-the-line software-based commercial and research systems [1], [4], [42], [43], but without the need for any external device that houses the ultrasound front-end and provides the cable connector to connect the probe. The LIGHTPROBE provides true continuous RF access $(\geq 240 \mathrm{Mb} / \mathrm{s} \text { per channel })^{2}$, which is comparable to the capabilities of the 256-vantage system $(206 \mathrm{Mb} / \mathrm{s}$ per channel) given its $6.6 \mathrm{~GB} / \mathrm{s}$ sustained data access [42]. Even though LiGHTPROBE supports only one fourth of the number of channels of these high-end systems, we are able to do so at a lower cost, power budget and smaller form factor.

Supporting ultrafast imaging at high frame-rates with a digital probe requires an elaborated power and thermal management as the peak power consumption in the probe may exceeds $10 \mathrm{~W}$ (VS13, $500 \mathrm{~Hz})$. Our thermal control management allows to safely use these modes with a defined periodicity.

Compared to the 16-channel (128 with multiplexing) digital ultrasound probe [14] equipped with USB 3.0 to connect to smartphones, our 64-channel LIGHTPROBE provides $4 \times$ more channels and even has a $30 \%$ lower overall probe power consumption $(5.84 \mathrm{~W}$ vs. $8.16 \mathrm{~W})$ for B-mode imaging (VS13 $50 \mathrm{~Hz})$. The aforementioned probe has a similar size $\left(180 \times 55 \times 35 \mathrm{~mm}^{3}\right)$ and performs the beamforming on the probe (FPGA) and the postprocessing on the smartphone's GPU. Under operation, the system heats up internally to $82.3^{\circ} \mathrm{C}$ and the transducer to $35^{\circ} \mathrm{C}$, allowing sustainable $\mathrm{B}$ Mode operation as the LightProbE does. Note that many digital probes not even supporting ultrafast imaging do not allow uninterrupted operation and require the probe to be periodically turned off for cooling. For example, the Sonon 300 series Wi-Fi probes (Healcerion, KOR) are specified for a maximal $10 \mathrm{~min}$ operation with $10 \mathrm{~min}$ resting time [40]. Clarius probes [18] can be equipped with an external fan for

${ }^{2} \mathrm{The} 240 \mathrm{Mb} / \mathrm{s}$ relate to the currently set sampling rate of $20 \mathrm{MS} / \mathrm{s}$.
TABLE III

COMPARISON WITH RELATED WORK

\begin{tabular}{lclll}
\hline System & ADC $^{\text {a Link }}$ & Mode & Connects to \\
\hline MobiUS PE & $?$ & USB & B & PC \\
Usan & 8 & USB & B & Dedicated Tablet \\
Visiq & $?$ & USB & B/M, Doppler & Dedicated Tablet \\
Lumify & $?$ & USB & B/M, Color Doppler & Smartphone/Tablet \\
iViz & $?$ & Cable & B, Color Doppler & Dedicated Tablet \\
Clarius & $?$ & Wi-Fi & B, Color Doppler & Smartphone/Tablet \\
Sonon300c & $?$ & Wi-Fi & B & Smartphone \\
Freestyle & $?$ & UWB & B, Color Doppler & Dedicated Device \\
\hline [15] & $64^{\text {b }}$ & Wi-Fi & SASB & - \\
[45] & $192^{\text {c }}$ & Wi-Fi & SASB (Vector Flow) & Tablet \\
[14] & 16 & USB 3.0 & B/C (MUX) & Smartphone \\
[46] & 1 & USB 2.0 & B (MSAS) & PC \\
[47] & 1 & Wi-Fi & B/3D & Display \\
\hline LightProbe & 64 & Optical & any (RF access) & PC \\
\hline DiPhAs [4] & 256 & PCIe & any (RF access) & PC \\
Vantage [42] & 256 & PCIe & any (RF access) & PC \\
\hline
\end{tabular}

a Number of ADC in commercial system rarely made public.

b System-level design study for a wireless probe.

c No probe hardware. Probe emulated with SARUS for demonstration.

d Smartphone \& tablet possible over USB/Wi-Fi.

longer scanning time or provide a docking station to cool probes back down to their operable temperature range.

Note that the LightPROBE hardware presented in this work has the required interfaces (USB, Wi-Fi) to connect to tablets and smartphones as well. Given the reduced data bandwidth of these interfaces, additional buffering or processing (beamforming) may be implemented on the LIGHTPROBE's FPGA. Implementing conventional digital beamforming (not ultrafast) is not critical from a power point of view: As shown in [44], powerful integrated digital beamformer (100-channel, 300 M focal points per second) consume a few hundreds of $\mathrm{mW}$, which is very little compared to the more than $2.0 \mathrm{~W}$ used in the front-end.

LighTPROBE is a prototype to demonstrate the capabilities an ultrafast digital probe may provide and to show how the main system-level challenges (interface speed, power/thermal management) can be solved. The current probe is built entirely with off-the-shelf components readily available. Thus there is a large improvement potential to reduce the size and power consumption if state-of-the-art components were used as described in recent literature: While the current $A F E$ is already very efficient (38.3 mW/cha @ 20 MS/s, measured), state-of-the-art literature systems can reach $38.6 \mathrm{~mW} / \mathrm{cha} @ 40 \mathrm{MS} / \mathrm{s}$ [20], which is a $2 \times$ energy efficiency increase in $\mathrm{mW} /$ cha per MS/s. These power figures are already reached by recent (2017) 32-channel AFEs (AFE58JD32, TI) consuming 42mW/ch @ $40 \mathrm{MS} / \mathrm{s}$. Our optical link uses a module consuming $0.87 \mathrm{~W}$ (a) $25 \mathrm{~Gb} / \mathrm{s}$. State-of-the-art modules like the ECUO Optical Firefly (Samtec) using VCSEL array technology can transfer $168 \mathrm{~Gb} / \mathrm{s}$ using $<1 \mathrm{~W}$ (TX) with a footprint of $2 \mathrm{~cm}^{2}$, resulting in a $6 \times$ higher link power efficiency and $7 \times$ size reduction. Similarly, the latest $16 \mathrm{~nm}$ Xilinx UltraScale+ FPGAs provide high-speed transceivers with twice the power efficiency $(50 \mathrm{~Gb} / \mathrm{s} @ 0.5 \mathrm{~W})$ and realize digital functionality with a $2.4 \times$ performance increase per Watt over the older $28 \mathrm{~nm}$ FPGA generation currently used in LIGHTPROBE. We estimate $1.2 \mathrm{~W}$ 
can be saved on the FPGA subsystem. By replacing the active $T / R$ switches with passive ones (e.g. MD0101, Microchip), another $0.73 \mathrm{~W}$ could be saved. The current $H V / L V$-supplies were highly optimized for footprint $\left( \pm 50 \mathrm{~V}\right.$ in $\left.9 \mathrm{~cm}^{2}\right)$ at the cost of good conversion efficiencies under low load. Currently, around half of the front-end power during power down modes (RX-STBY and RX-PDN) is lost in the converters, as these supplies were designed for high-efficiency at their nominal load. If all these changes were implemented, we estimate that the total consumption in RDY can be reduced by $2.4 \times$ from $8.08 \mathrm{~W}$ to $3.3 \mathrm{~W}$ (see Fig. 10 RIGHT). Considering this $2.4 \times$ possible reduction, we expect even HFR imaging (VS13, $500 \mathrm{~Hz}$ ) can be operated with a total probe consumption of around $6 \mathrm{~W}$. The power consumption and size may be reduced even further if the FPGA is replaced by a application-specific integrated-circuit (ASIC) that co-integrates part of the transmit and receive path with the transceivers for the optical link.

The adapter that allows connecting the optical link of the LightPRoBE to the PC is currently implemented with a commercial FPGA Development Board (Xilinx KCU105, $3000 \$$ ) plugged into a PCIe slot. This solutions can easily be replaced with a more cost-efficient solution: Changing the protocol of the fiber link from Aurora to Ethernet allows using an off-the-shelf server-grade QSFP Ethernet network card on the PC side. This brings the adapter cost down to 300-400\$.

The LIGHTPROBE is larger compared to commercial probes. Given that the PCBs are only sparsely populated (Fig. 3) and the previously mentioned improvements, we expect that a simple PCB re-layout would reduce the probe length by $2 \times$. By switching to the latest fully-integrated TX chips such as the STHV1600 (STM) the TX boards are no longer needed as TXBF, Pulser and T/R can be implemented with 4 chips using the same space only the T/R switches need now. This halves the probe thickness, resulting in a size comparable with current probes. As smaller probes can dissipate less heat, any reduction in size must go in hand with the previously mentioned power reductions in order to keep the performance level of the probe.

\section{CONClusions}

We have presented a 64-channel digital ultrasound transducer probe (LIGHTPROBE), which confines the entire ultrasound front-end in the probe handle and outputs the raw data samples in real-time over a high-speed optical link. With LightPROBE, very cost-effective yet versatile and powerful ultrasound systems can be built by connecting the probe to a commodity device (PC) performing the processing in software by running the required ultrasound application.

The LightPROBE not only supports ultrafast imaging, it also exploits its ultrafast capabilities to reduce the power consumption during normal-rate B-mode imaging with smart duty-cycling of the front-end. A novel thermal management approach allows the LIGHTPROBE to exploits its thermal capacitance to supports intermittent operation of highperformance modes whose power dissipation exceeds the sustainable passive-cooling capabilities. Our thermal management ensures periodic activation of these modes without ever having to turn off the probe of for cooling, providing a consistent quality of service to the operator.

Our work shows that using digital probes instead of analog probes is a feasible system architecture option for future cart-based software-defined ultrasound systems, as they allow to build powerful and cost-efficient systems thanks to the minimal amount of ultrasound specific hardware required. Also, our approach to increase the number of receive channels to support ultrafast imaging with the goal of reducing the average front-end power consumption may be leveraged in future mobile systems to support more modalities.

\section{ACKNOWLEDGMENT}

We thank Manfred Moses, Christian Risser, Daniel Speicher, Christian Degel and Peter-Karl Weber from IBMT and Alfonso Blanco from Microelectronics Design Center and Matthias Brägger to make this project possible. This work is supported by the projects LIGHTPROBE and BIODEV funded by NanoTera.ch with Swiss Confederation financing.

\section{REFERENCES}

[1] C. H. Alfred et al., "Ultrasound Open Platforms for Next-Generation Imaging Technique Development," IEEE Trans. Ultrason., Ferroelectr. Freq. Control., vol. 65, no. 7, pp. 1078-1092, 2018.

[2] H. K. So et al., "Medical ultrasound imaging: To GPU or not to GPU?" IEEE Micro, vol. 31, no. 5, pp. 54-65, 2011.

[3] B. Y. S. Yiu, I. K. H. Tsang, and A. C. H. Yu, "GPU-based beamformer: Fast realization of plane wave compounding and synthetic aperture imaging," IEEE Trans. Ultrason., Ferroelectr., Freq. Control., vol. 58, no. 8, pp. 1698-1705, 2011.

[4] C. Risser et al., "High Channel Count Ultrasound Beamformer System with External Multiplexer Support for Ultrafast 3D / 4D Ultrasound," in IEEE Ultrasonics Symposium Proceedings, 2016.

[5] A. Kurth et al., "Mobile ultrasound imaging on heterogeneous multicore platforms," in Proceedings of the 14th ACM/IEEE Symposium on Embedded Systems for Real-Time Multimedia, 2016.

[6] J. Ma et al., "Ultrasound phase rotation beamforming on multi-core DSP," Ultrasonics, vol. 54, no. 1, pp. 99-105, 2014.

[7] A. Agarwal et al., "Single-chip solution for ultrasound imaging systems: Initial results," Proceedings - IEEE Ultrasonics Symposium, pp. 1563 1566, 2007.

[8] J. Bercoff, Ultrafast Ultrasound Imaging, O. Minin, Ed. InTech, 2011.

[9] M. Tanter and M. Fink, "Ultrafast imaging in biomedical ultrasound," IEEE Trans. Ultrason., Ferroelectr., Freq. Control., vol. 61, no. 1, pp. $102-119,2014$.

[10] C. A. Villagomez Hoyos et al., "Accurate Angle Estimator for HighFrame-Rate 2-D Vector Flow Imaging," IEEE Transactions on Ultrasonics, Ferroelectrics, and Frequency Control, vol. 63, no. 6, pp. 842-853, 2016.

[11] S. Holbek et al., "Real-time 2-D Phased Array Vector Flow Imaging," IEEE Transactions on Ultrasonics, Ferroelectrics, and Frequency Control, vol. 65, no. 7, pp. 1205-1213, 2018.

[12] M. Correia et al., "Ultrafast Harmonic Coherent Compound (UHCC) Imaging for High Frame Rate Echocardiography and Shear-Wave Elastography," IEEE Transactions on Ultrasonics, Ferroelectrics, and Frequency Control, vol. 63, no. 3, pp. 420-431, 2016.

[13] O. M.-G. D. Romero-Laorden, J. Villazón-Terrazas and A. Ibez, "Strategies for Hardware Reduction on the Design of Portable Ultrasound Imaging Systems," Advancements and Breakthroughs in Ultrasound Imaging, pp. 243-268, 2013.

[14] S. Ahn et al., "Smartphone-based Portable Ultrasound Imaging System: Prototype Implementation and Evaluation," in IEEE Ultrasonics Symposium Proceedings, vol. 1, 2015, pp. 1-4.

[15] T. Di Ianni et al., "System-level Design of an Integrated Receiver Frontend for a Wireless Ultrasound Probe," IEEE Transactions on Ultrasonics, Ferroelectrics, and Frequency Control, vol. 63, no. 11, pp. 1935-1946, 2016.

[16] Philips Healthcare, "Philips VISIQ Brochure," Tech. Rep., 2014.

[17] Philips Healthcare, "Lumify Ultrasound System," Tech. Rep., 2017. 
[18] Clarius Mobile Health Corporation, "Clarius Ultrasound Scanner User Manual,” Tech. Rep., 2017.

[19] International Electrotechnical Commission, "International Standard IEC 60601-1," pp. 563-564, 2005.

[20] E. Roa, "A 16-channel 38.6 mW / ch Fully Integrated Analog Front-end for Handheld Ultrasound Imaging," in Biomedical Circuits and Systems Conference (BioCAS), 2014 IEEE, 2014, pp. 6-9.

[21] C. Chen et al., "A Front-End ASIC for Miniature 3-D Ultrasound Probes with In-Probe Receive Digitization," in Ultrasonics Symposium (IUS), 2017 IEEE International, 2017, pp. 7-10.

[22] T. Di Ianni et al., "Analog gradient beamformer for a wireless ultrasound scanner," SPIE Medical Imaging, vol. 9790, no. April 2016, p. 979010 , 2016.

[23] H. Hewener and S. Tretbar, "Mobile ultrafast ultrasound imaging system based on smartphone and tablet devices," 2015 IEEE International Ultrasonics Symposium, IUS 2015, pp. 1-4, 2015.

[24] P. A. Hager et al., "LightProbe: A 64-Channel Programmable Ultrasound Transducer Head with an Integrated Front-end and a $26.4 \mathrm{~Gb} / \mathrm{s}$ Optical Link," in 2017 IEEE International Symposium on Circuits and Systems (ISCAS), 2017, pp. 302-305.

[25] P. P. A. Hager et al., "UltraLight: An Ultrafast Imaging Platform based on a Digital 64-Channel Ultrasound Probe," in IEEE Ultrasonics Symposium Proceedings, 2017.

[26] C. Kachris and I. Tomkos, "A survey on optical interconnects for data centers," IEEE Communications Surveys and Tutorials, vol. 14, no. 4, pp. 1021-1036, 2012.

[27] G. Montaldo et al., "Coherent plane-wave compounding for very high frame rate ultrasonography and transient elastography," IEEE Transactions on Ultrasonics, Ferroelectrics, and Frequency Control, vol. 56, no. 3, pp. 489-506, 2009.

[28] J. Jensen, M. Stuart, and J. Jensen, "Optimized plane wave imaging for fast and high quality ultrasound imaging," IEEE Transactions on Ultrasonics, Ferroelectrics, and Frequency Control, vol. 63, no. 11, pp. 1922-1934, 2016.

[29] G. Frey and R. Chiao, "4Z1c Real-Time Volume Imaging Transducer," Siemens Healthcare, Tech. Rep., 62008.

[30] J. A. Jensen et al., "Synthetic aperture ultrasound imaging," Ultrasonics, vol. 44, Supple, pp. e5 - e15, 2006.

[31] C. Frazier and J. O'Brien, W.D., "Synthetic aperture imaging with a virtual source element," 1996 IEEE Ultrasonics Symposium. Proceedings, vol. 2, no. 1, pp. 196-207, 1996.

[32] S. Nikolov and J. A. Jensen, "Virtual ultrasound sources in highresolution ultrasound imaging," Proc. SPIESPIE, vol. 4687, pp. 4687 - $4687-11,2002$.

[33] R. Y. Chiao and X. Hao, "Coded excitation for diagnostic ultrasound: A system developer's perspective," IEEE Transactions on Ultrasonics, Ferroelectrics, and Frequency Control, vol. 52, no. 2, pp. 160-170, 2005.

[34] I. Trots, Y. Tasinkevych, and A. Nowicki, "Orthogonal golay codes with local beam pattern correction in ultrasonic imaging," IEEE Signal Processing Letters, vol. 22, no. 10, pp. 1681-1684, 2015.

[35] T. L. Szabo, Diagnostic Ultrasound Imaging: Inside Out (Second Edition). Boston: Academic Press, 2014.

[36] H. Liebgott et al., "Plane-Wave Imaging Challenge in Medical Ultrasound," IEEE International Ultrasonics Symposium, IUS, vol. 2016Novem, 2016.

[37] P. A. Hager, P. A. Jud, and L. Benini, "A Low-Cost Software-Defined Ultrasound System Capable of High-Speed Ultrasound Bubble Tracking," in IEEE Ultrasonics Symposium Proceedings, 2018, p. [To Appear].

[38] Signostics, "Uscan - User Manual," Tech. Rep. November, 2017.

[39] Siemens Healthcare, "ACUSON Freestyle Datasheet," Tech. Rep., 2013.

[40] Healcerion, "Sonon ultrasound imaging system 300c," Tech. Rep., 2016.

[41] Sonosite, "iViz User Guide," Tech. Rep., 2016.

[42] Verasonics, "Vantage Family Brochure," Tech. Rep., 2016.

[43] J. A. Jensen et al., "SARUS: A synthetic aperture real-time ultrasound system," IEEE Trans. Ultrason., Ferroelectr., Freq. Control., vol. 60, no. 9, pp. 1838-1852, 2013.

[44] P. A. Hager, A. Bartolini, and L. Benini, "Ekho: A 30.3W, 10k Channels Fully-Digital Integrated 3D Beamformer for Medical Ultrasound Imaging Achieving 298.1M Focal Points per Second," IEEE Transactions on Very Large Scale Integration (VLSI) Systems, vol. 24, no. 5, pp. 19361949, 2015

[45] T. D. Ianni et al., "A Vector Flow Imaging Method for Portable Ultrasound Using Synthetic Aperture Sequential Beamforming," IEEE Transactions on Ultrasonics, Ferroelectrics and Frequency Control, vol. 64, no. 11, pp. 1655-1665, 2017.
[46] T. L. Van Den Heuvel et al., "Development of a Low-Cost Medical Ultrasound Scanner Using a Monostatic Synthetic Aperture," IEEE Transactions on Biomedical Circuits and Systems, vol. 11, no. 4, pp. 849-857, 2017

[47] M. R. Sobhani et al., "Portable low cost ultrasound imaging system," IEEE International Ultrasonics Symposium, IUS, vol. 2016-Novem, no. $113,2016$.

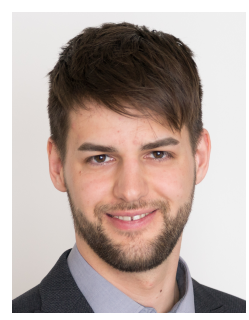

Pascal Alexander Hager received his M.Sc. degree with distinction in electrical engineering and information technology from ETH Zurich, Switzerland in 2014. Since then, he has been with the Integrated Systems Laboratory (IIS), ETH Zurich, where he is currently pursuing his Ph.D. degree. His research interests include medical ultrasound imaging, digital signal processing and low-power integrated circuit design. Mr. Hager received the Best Paper Award at the 2013 IEEE VLSI-SoC Conference.

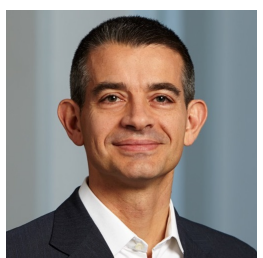

Luca Benini holds the chair of digital circuits and systems at ETH Zurich and is Full Professor at the Universita di Bologna. Dr. Benini's research interests are in energy-efficient system design for embedded and high-performance computing. He is also active in the area of energy-efficient smart sensors and ultra-low power VLSI design. He has published more than 800 papers and five books. He is a Fellow of the IEEE and the ACM and a member of the Academia Europaea. He is the recipient of the 2016 IEEE CAS Mac Van Valkenburg award. 